\title{
Presence of varicella zoster virus in zosteriform leukaemia cutis
}

\author{
E. Szlávicz, ${ }^{1}$ E. Kálmán, ${ }^{1}$ C. Gyömörei, ${ }^{2}$ L. A. Kovács, ${ }^{1}$ H. Ócsai, ${ }^{2}$ E. Varga, ${ }^{2}$ J. Oláh, ${ }^{2}$ R. Gyulai ${ }^{1}$ and \\ Z. Lengyel ${ }^{1}$ \\ ${ }^{1}$ Department of Dermatology, Venereology and Oncodermatology, University of Pecs, Pecs, Hungary; and 2Department of Dermatology and Allergology, \\ University of Szeged, Szeged, Hungary \\ doi:10.1111/ced.13899
}

\section{Summary}

\begin{abstract}
Leukaemia cutis is a relatively rare manifestation in chronic lymphocytic leukaemia, characterized by a diverse morphology of skin lesions. We report two patients who developed zosteriform skin symptoms; however, the histological analysis revealed leukaemia infiltration as the cause of their symptoms. Contrary to previous reports, varicella zoster virus DNA was detectable in the lesions. These findings suggest that varicella zoster virus plays an active role in the development of zosteriform leukaemia cutis.
\end{abstract}

Chronic lymphocytic leukaemia (CLL) is one of the most common haematological disorders in elderly people. Detection of the disease is often an incidental finding during unrelated laboratory tests, although classic B symptoms (weight loss, fever, night sweats) may also indicate the development of CLL. The most prevalent symptoms primarily include: enlargement of the lymph nodes, hepatosplenomegaly and consequences of bone marrow suppression-related deficiencies (anaemia, leucopenia, thrombocytopenia). ${ }^{1}$

CLL-associated skin symptoms can be classified into specific and nonspecific lesions: the former are characterized as skin manifestations of a haematological disorder, while the latter are the results of impaired immune system functionality. ${ }^{2}$ Infiltration of the skin by myeloid/lymphoid tumour cells is defined as leukaemia cutis, which may be present in several morphological forms, including papules, vesicles, blisters, infiltrated nodules, and ulcerated or necrotic lesions. ${ }^{3,4}$ Cutaneous involvement is generally related to T-cell derived haematological malignancies, but approximately $4-20 \%$ of B-cell CLL cases also exhibit leukaemia cutis. ${ }^{3-5}$

Correspondence: Dr Eszter Szlávicz, Department of Dermatology, Venereology and Oncodermatology, University of Pecs, 1 Akac Street, HU-7632, Pecs, Hungary

E-mail: szlavicz.eszter@gmail.com

Conflict of interest: the authors declare that they have no conflicts of interest.

Accepted for publication 9 October 2018
We report two patients with leukaemia cutis with positive PCR findings for varicella zoster virus (VZV).

\section{Report}

Patient 1 was a 71-year-old woman with a 4-year history of CLL. She had received rituximab and chlorambucil therapy due to disease progression. Notably, 3 months following this treatment, zoster-like eruptions formed on the right side of her upper torso. The patient's skin symptoms did not respond to oral aciclovir (400 mg five times/day, prescribed at the Department of Haematology). Several weeks following use of aciclovir novel symptoms developed on the patient's nose and at the hairline.

On admission, several necrotic papules were observed on the right side of the patient's upper torso and head, in a zosteriform arrangement. Lesions of the forehead and nape of the neck were similar in appearance. On the nose, a large centrally necrotic papule was seen, resembling keratoacanthoma (Fig. 1a-d). Laboratory investigations revealed alterations characteristic of CLL. Histological samples were taken from the zosteriform lesions of the upper torso and the keratoacanthoma-like lesion upon the nose. Intravenously, aciclovir was initiated at $8 \mathrm{mg} / \mathrm{kg}$ three times daily. Histopathological analysis of both the zosteriform and the keratoacanthoma-like lesions showed a dense, small lymphocytic infiltration indicating cutaneous manifestations of CLL (Fig. 1). Samples obtained from the tumours were also tested for VZV DNA by 

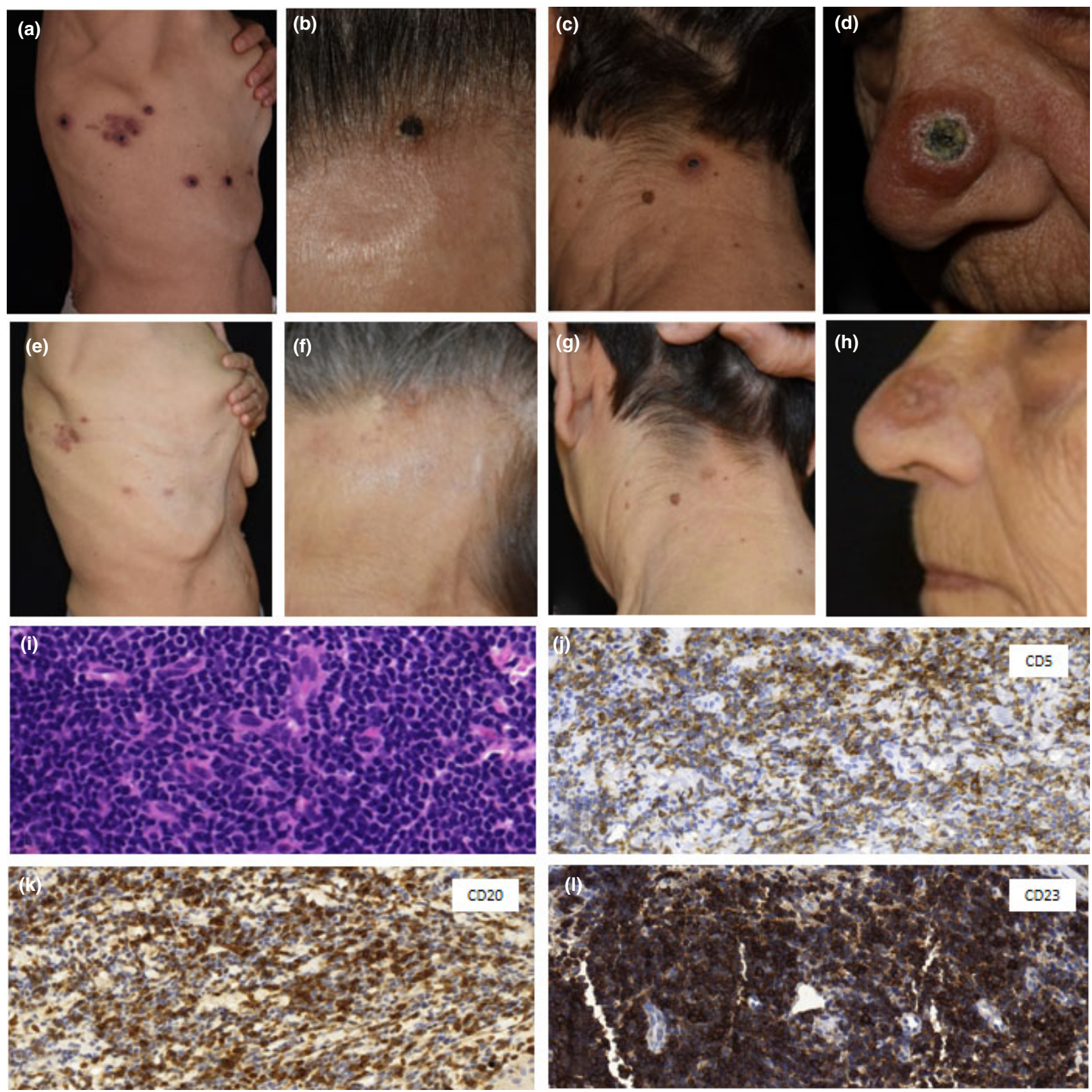

Figure 1 Patient 1 with chronic lymphocytic leukaemia. (a-c) Necrotic papules in a zosteriform arrangement on (a) the right side of the chest wall, (b) nape of the neck, (c) and forehead; (d) morphologically different lesion on the tip of the nose, which was centrally retracted and covered by a necrotic crust. (e-g) Following chlorambucil treatment, there was marked regression of the skin symptoms: (e) on the chest, only hyperpigmented macules were observable, while the lesions on (f) the nape and (g) forehead were almost completely healed. (h) There was a residual erythematous papule on the nose, and the previous necrotic crust had disappeared. (i) Histological examination depicts dense, dermal infiltrates of small and medium sized lymphocytes in all samples (haematoxylin and eosin, original magnification $\times 400)$. $(\mathrm{j}-\mathrm{l})$ Immunohistochemistry showed the infiltrates to be positive for (j) CD5, (k) CD20 and (l) CD23. Original magnification $(\mathrm{j}-\mathrm{l}) \times 400$.

PCR, and despite 2 months of prior antiviral therapy, VZV DNA was detected in the necrotic infiltrates.

The patient was transferred to the Haematology ward, in which chlorambucil therapy was initiated and antiviral treatment was continued. The VZV symptoms showed slow regression, and 3 months following hospitalization, only hyperpigmented macules were present on the upper torso, forehead and 
nape, while the lesion on the nose also showed marked improvement (Fig. 1e-h). However, despite the skin symptoms improving and the chlorambucil being continued, the CLL continued to progress. The patient died owing to respiratory insufficiency 3 months after the final cycle of chemotherapy (7 months after the appearance of zosteriform lesions).
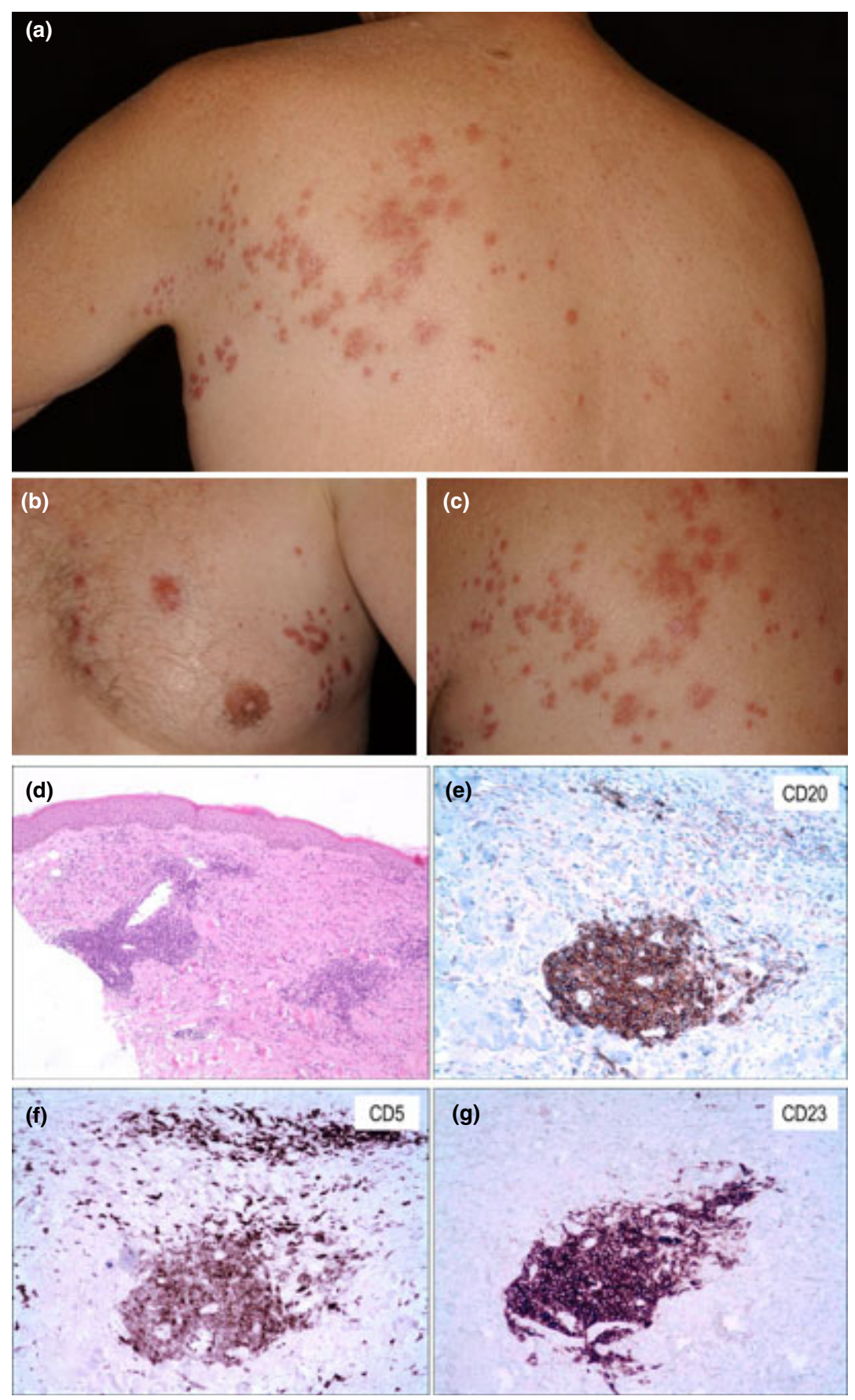

Figure 2 Patient 2 with chronic lymphocytic leukaemia. (a-c) Infiltrated papules localized to the left thoracic area (Th1/2 dermatomes). (d) Histological examination depicts dense, dermal infiltrates of small and medium sized lymphocytes in all samples (haematoxylin and eosin, original magnification $\times 100)$. $(\mathrm{e}-\mathrm{g})$ Immunohistochemistry showed the infiltrates to be positive for (e) CD5, (f) CD20 and (g) CD23 (original magnification $\times 200$ ). 
Patient 2 was a 49-year -old man with 5 years of disease development, who was treated with fludarabine/ cyclophosphamide chemotherapy because of recurrent lymphadenopathy. Following two cycles of treatment, zosteriform cutaneous lesions developed upon the patient's back. Despite 2 months of antiviral treatment (oral aciclovir $800 \mathrm{mg}$ five times/day, followed by $400 \mathrm{mg}$ twice daily), painful, red papules were still observable in a dermatomal arrangement (Fig. 2a-c).

Histological analysis of the skin biopsy revealed infiltration of CLL with necrosis and granulomatous inflammation. The possibility of cutaneous metastases was supported by immunohistochemical staining, with mononuclear cells showing overexpression of CD5 and CD20 (Fig. 2d-g). Similar to patient 1, VZV DNA was also detectable in the lesions.

Following the diagnosis of cutaneous CLL lesions, the patient received one additional cycle of fludarabine/cyclophosphamide, but died from pneumonia 1 month following the final round of chemotherapy (9 months after the formation of skin symptoms).

To date, very few articles have been published highlighting zosteriform skin symptoms in patients with CLL. These symptoms can be divided into nonspecific (such as granulomatosus reactions, purpura and cutaneous vasculitis) and specific (tumorous) lesions. ${ }^{1,3,6}$ Regarding specificity in the occurrence of lesions, it is not clear whether the presence of tumorous cells in the skin should be considered a negative prognostic factor. $^{1,2,4,7}$ Occasionally, skin lesions are triggered by antigenic stimulation, a phenomenon usually not associated with disease progession. ${ }^{1,2,4,8}$ In contrast, infiltration of large cells with features of centroblasts and/ or immunoblasts is often a negative prognostic sign. ${ }^{1,9}$ Although no large cells were observable in our skin samples, both patients died within a year (7 and 9 months, respectively) following skin infiltration. Even though a relatively short time elapsed between the presence of the cutaneous manifestation and the patients' deaths, there is insufficient evidence that would support a causal association between the presence of cutaneous CLL manifestation and prognosis.

The role of preceding viral infection in tumorous infiltration is not completely understood. Most reports have been published on patients with herpes zoster. There are several theories on the zosteriform arrangement of tumour cells. Tumour cells may spread along the neurons, or mediators released from damaged nerves/keratinocytes may attract lymphoid cells to the skin. $^{5,6,8}$ Moreover, antigenic stimuli may not only recruit healthy, but also malignant cells. ${ }^{1,2,4}$ Several studies point to the often referred Wolf isotopic response, in which a new skin disorder develops within the scars and/or tissue from a previous, unrelated disease. ${ }^{6}$ Interestingly, the zosteriform arrangement can be a feature of other tumours, such as mammalian and colon adenocarcinomas, or metastatic melanoma. ${ }^{4}$

Detectability of VZV in lesional samples is also the subject of extensive discussion. While most studies in which VZV detection was attempted by PCR originating from zosteriform skin lesions yielded negative results, ${ }^{4,5,7,8}$ there are several publications in which VZV DNA appears in skin lesions. ${ }^{10}$ Moreover, the presence of herpes simplex DNA was also described in cutaneous manifestations of CLL. ${ }^{2}$

In conclusion, we report two cases of zosteriform cutaneous infiltration. Our results indicate that the presence of VZV plays an important factor in the development and maintenance of cutaneous infiltrates. In contrast to previous reports, the cutaneous lesions in our patients showed distinct VZV DNA positivity, suggesting that VZV is implicated in immunological processes leading to leukaemia cutis.

\section{Acknowledgements}

We thank L. Krenács and E. Bagdi for immunohistological analysis and M. Kiss for VZV PCR detection.

\section{Learning points}

- Two patients with CLL developed zosteriform lesions.

- Contrary to previous reports, it was shown that VZV DNA may be detectable in the cutaneous involvement of CLL.

- VZV may likely be an active participant in the cutaneous infiltration of neoplastic lymphoid cells.

- It is unclear whether a disease-specific cutaneous infiltration should be considered as a negative prognostic factor.

\section{References}

1 Hapgood G, Mooney E, Dinh HV et al. Leukaemia cutis in chronic lymphocytic leukaemia following varicella zoster virus reactivation. Intern Med J 2012; 42: 1355-8.

2 Ziemer M, Bornkessel A, Hahnfeld S, Weyers W. 'Specific' cutaneous infiltrate of B-cell chronic lymphocytic 
leukemia at the site of a florid herpes simplex infection. J Cutan Pathol 2005; 32: 581-4.

3 Agnew KL, Ruchlemer R, Catovsky D et al. Cutaneous findings in chronic lymphocytic leukaemia. $\mathrm{Br}$ J Dermatol 2004; 150: 1129-35.

4 Antunes J, Pacheco D, Travassos R, Filipe P. Zosteriform B-cell chronic lymphocytic leukemia infiltration. Dermatol Online J 2011; 17: 9.

5 Elgoweini M, Blessing K, Jackson R et al. Coexistent granulomatous vasculitis and leukaemia cutis in a patient with resolving herpes zoster. Clin Exp Dermatol 2011; 36: 749-51.

6 Jaka-Moreno A, Lopez-Pestana A, López-Núñez M et al. Wolf's isotopic response: a series of 9 cases. Actas Dermosifiliogr 2012; 103: 798-805.
7 Cerroni L, Zenahlik P, Kerl H. Specific cutaneous infiltrates of B-cell chronic lymphocytic leukemia arising at the site of herpes zoster and herpes simplex scars. Cancer 1995; 76: 26-31.

8 Moreira E, Lisboa C, Azevedo F et al. Postzoster cutaneous pseudolymphoma in a patient with B-cell chronic lymphocytic leukaemia. J Eur Acad Dermatol Venereol 2007; 21: 1112-14.

9 Cerroni L, Zenahlik P, Höfler G et al. Specific cutaneous infiltrates of B-cell chronic lymphocytic leukemia: a clinicopathologic and prognostic study of 42 patients. Am J Surg Pathol 1996; 8: 1000-10.

10 Doutre MS, Beylot-Barry M, Beylot C et al. Cutaneous localization of chronic lymphocytic leukemia at the site of chickenpox. J Am Acad Dermatol 1997; 36: 98-9. 